\title{
Variable Speed of Light with Time and General Relativity
}

\author{
Giuseppe Pipino \\ Technical High School, ITIS Malignani, Udine, Italy \\ Email: giupipino@libero.it
}

How to cite this paper: Pipino, G. (2021) Variable Speed of Light with Time and General Relativity. Journal of High Energy Physics, Gravitation and Cosmology, 7, 742-760. https://doi.org/10.4236/jhepgc.2021.72043

Received: February 8, 2021

Accepted: April 27, 2021

Published: April 30, 2021

Copyright $\odot 2021$ by author(s) and Scientific Research Publishing Inc. This work is licensed under the Creative Commons Attribution International License (CC BY 4.0).

http://creativecommons.org/licenses/by/4.0/

\begin{abstract}
In a previous article entitled: "Evidences for varying speed of light with time" [1], a series of observational evidence was presented in favor of the hypothesis that the speed of light varies with time according to the relationship $\mathrm{d} c / \mathrm{d} t=$ $-H c$, where $H$ is the Hubble constant which is considered a universal constant. In this paper we propose to elaborate on the observational evidence supporting the hypothesis, and to probe the consequences of this relationship on General Relativity. Also we will provide a theoretical justification of the previous relationship and we will show how from it we can deduce galactic velocity curves. We can deduce the important empirical Tully-Fisher relation linking these curves to the baryonic mass of the galaxy and we can justify the apparent accelerated expansion of the universe without intervening elusive entities such as dark matter and dark energy.
\end{abstract}

\section{Keywords}

Abnormal Acceleration, Modified Newtonian Dynamic, Tully-Fisher Relation, Redshift, Hubble's Law, Dark Matter, Dilation of Light Curves of Supernovae Ia, Acceleration of Expansion of Universe, General Relativity

\section{Observational Evidences in Favor of Time-Varying $c$}

The hypothesis of variable speed of light with time is capable to provide both a qualitative and quantitative justification for various observational phenomena that have not, at the moment, obtained a satisfactory explanation, not even in the relativistic field. Among these

1) In the past, many spacecrafts (Pioneer 10, Pioneer 11, Galileo, Ulysses) [2] have been sent into space.

These spacecrafts have been equipped with sources of electromagnetic radiation with frequency $f_{e}$. By comparing the frequency $f_{0}$ of the received signal with the frequency $f_{e}$ of an identical source placed in the terrestrial laboratory, it was 
possible to determine the speed of the spacecraft itself with the formula of the non-relativistic Doppler effect, which in the case of a receding source provides: $f_{0}=f_{e}(1-v / c)$.

Hence $\left(f_{0}-f_{e}\right) / f_{e}=-v / c$.

The negative sign indicates that the observed frequency is smaller than the emitted frequency.

It was noted in this way that all the spacecrafts sent into space that have left the solar system, instead of proceeding at a constant speed (since at the distances reached the gravitational attraction from the Sun is negligible) seem to be subject to a deceleration $a_{c}=-(8 \pm 3) \times 10^{-10} \mathrm{~m} \cdot \mathrm{sec}^{-2}$, in the direction of the joining spacecraft-Sun and directed towards the Sun, which remains CONSTANT throughout the observation time and which is the same for all spacecrafts observed.

The VSLT hypothesis explains this deceleration (called anomalous because no convincing explanations have been found for it) by stating that the variation $\Delta f f f_{0}$ is not due to the deceleration of the spacecraft, but is due to the fact that the signal used to establish the speed is subject to a variation $\mathrm{d} c \mathrm{~d} t=-H c$ which is reflected in an identical frequency variation.

This explains why the (apparent) deceleration observed for all spacecrafts is always the same and explains why this deceleration does not vary over time.

A calculation of the deceleration $a_{c}=-H c$, assuming for $H$ (Hubble constant) the value $H=69 \mathrm{~km} \cdot \mathrm{sec}^{-1} \cdot \mathrm{Mpc}^{-1}=2.3 \times 10^{-18} \mathrm{sec}^{-1}$ and for $c$ the currently measured value for the speed of light $\left(c=c_{0}=3 \times 10^{8} \mathrm{~m} \cdot \mathrm{sec}^{-1}\right)$ yields the value $a_{c} \approx-7 \times 10^{-10} \mathrm{~m} \cdot \mathrm{sec}^{-2}$ which is compatible with the observed value.

2) The second effect that VSLT theory is capable to explain is the anomalous rotation curve of the galaxies.

The stars of a galaxy revolve around their nucleus (each galaxy is thought to have a supermassive black hole in its center, in which most of the galactic mass is concentrated) with a motion that, according to Newtonian mechanics, is approximately circular.

If $m=$ star mass, $M=$ nucleus mass, we can equalize the gravitational force of attraction of the nucleus $F=G M m / r^{2}$ with the centrifugal force acting on the star $F=m v^{2} / r$, obtaining $v^{2}=G M / r$.

The trend of the star's rotation speed should therefore tend to zero as the distance from the galactic center increases. Actually, as the distance increases, the speed of the stars does not decrease to zero, but beyond a certain value $r_{\text {crit }}$ this speed remains almost constant.

Astrophysicists have tried to explain this phenomenon using the hypothesis of "dark matter", [3] or the hypothesis that every galaxy is surrounded by a halo of invisible matter that causes the gravitational potential to have a trend such as to produce the velocity curve observed.

The characteristics of dark matter should be very particular, since this matter should be completely transparent to radiation, but at the same time produce gravitational effects.

The particles that should make up dark matter (non-baryonic) have been 
called WIMPS and, although have been researched for many decades, they have never been observed. The hypothesis of dark matter has not been abandoned, but rather it has become dominant. The main reason is that this hypothesis allows to give an answer to a very serious cosmological problem posed by General Relativity. That is, the GR provides a so-called critical value for the density of matter of the universe. If the universe had this density, its geometry should be flat.

Now many measurements (including those performed on the cosmic background radiation) indicate that the universe has a flat geometry, however the density of matter that results by adding the mass of all visible galaxies is much lower than the critical value. Dark matter resolves this divergence between the theoretical value of density and the experimental one.

\section{Modificated Newtonian Dynamic (MOND)}

An attempt to explain velocity curves apart from dark matter was made by physicist M. Milgrom [4]. He hypothesized that Newton's law $F=$ ma only holds for values of $a>a_{0}$, being $a_{0}$ an extremely small value of acceleration to which Milgrom, assuming $H=50 \mathrm{~km} \cdot \mathrm{sec}^{-1} \cdot \mathrm{Mpc}^{-1}$, attributed the value $a_{0}=2 \times 10^{-10}$ $\mathrm{m} \cdot \mathrm{sec}^{-2}$.

The law postulated by Milgrom in substitution of the second law of Newton is $F=\mu m a$, being $\mu$ a coefficient that depends on the acceleration and that is equal to 1 in the normal case in which $a \gg a_{0}$, while it is $\mu=a / a_{0}$ in the case of very small values, i.e. falling below the value $a_{0}$.

We note that Milgrom's strange hypothesis produces the desired result.

Indeed if $a<a_{0}$ then $F=\mu m a=m a^{2} / a_{0}$. Equating with gravitational force $F=$ $G M m / r^{2}$ we find $a^{2} / a_{0}=G M / r^{2}$, but $a=v^{2} / r$. Then $v^{4} /\left(a_{0} r^{2}\right)=G M / r^{2}$, that is

$$
v=\sqrt[4]{a_{0} G M}
$$

As we can see the speed of the stars that are at a great distance from the nucleus so their centripetal acceleration is less than the constant $a_{0}$, remains constant.

Milgrom's theory, known as MOND (Modified Newtonian Dynamic) is capable to predict the velocity curves of galaxies based on their mass $\mathrm{M}$ (normal or baryonic matter) regardless of the existence of a phantomatic dark matter.

\section{Tully-Fisher Experimental Relation}

Milgrom's theory is confirmed by the experimental observations of Tully-Fisher [5] which link the baryon mass, including gas clouds, of galaxies with the asymptotic value of velocity obtained from the velocity curves (Figure 1). In this figure, $M *$ indicates the mass value of the galaxy (in units of solar masses), while $V_{c}$ indicates the asymptotic value of the velocity curves (in $\mathrm{Km} / \mathrm{s}$ ). Despite the agreement with the experimental data, Milgrom's theory was not successful for several reasons. First, the starting hypothesis, namely the modification of the Newtonian dynamics seems quite arbitrary. The same value of the constant $a_{0}$ is arbitrary. 


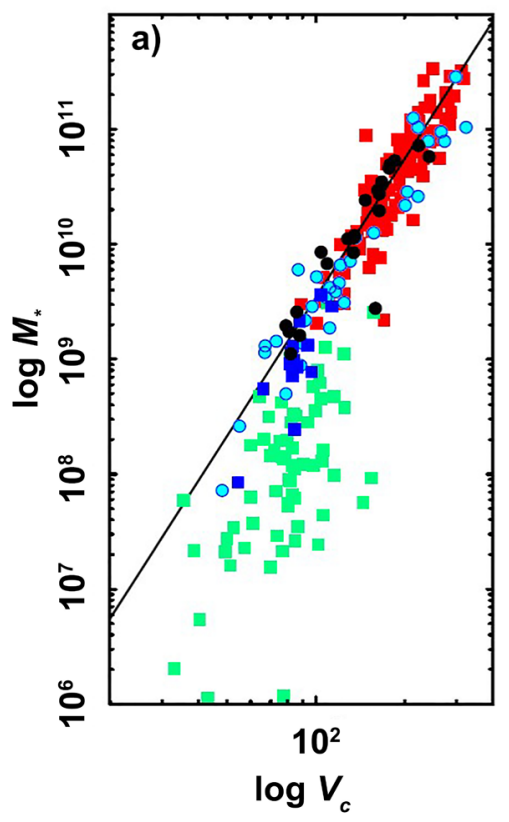

Figure 1. Mass of galaxies (in units of solar masses) as a function of asymptotic velocity curves (in $\mathrm{km} / \mathrm{s}$ ). The scale of the axes is logarithmic. The line shown interpolates the data for spiral galaxies.

Furthermore, MOND foresees an exactly constant velocity trend in the case in which $a<a_{0}$. Conversely, the light curves reveal a trend of $v$ with the distance of a parabolic type as shown in Figure 2. But the main reason, as mentioned, is that the hypothesis of a dark matter halo surrounding every galaxy helps to solve the problem of the flatness of the universe.

\section{VSLT Theory and Galactic Velocity Curve}

The explanation that VSLT theory provides for velocity curves is very simple. This explanation does not require any modification of Newtonian mechanics nor does it require the existence of dark matter.

When detecting the rotation speed of a star around the galactic center, the measurement of the frequency shift $\Delta f f$ of the signals emitted by the star is used. The observed shift is connected to the rotation speed by $|\Delta f| f \mid=v / c$.

The $\Delta f f$ ratio is taken as an absolute value because its sign depends on whether the source is approaching or moving away.

Actually, the observed shift not only measures the rotation speed of the star but also measures the deceleration that the light rays coming from the star undergo, due to the fact that the instant $t_{e}$ of emission of the signal and the instant of the observation $t_{0}$, are very different and, in this whole interval, the speed of light, emitted by atoms at different times, has changed.

It is important to point out that, for VSLT theory, the speed of light does not vary during the journey from the source to the observer. In other words, empty space is really "empty" and does not exert any braking action on photons. What changes is the speed of light emitted by atoms in different times. In other words, 


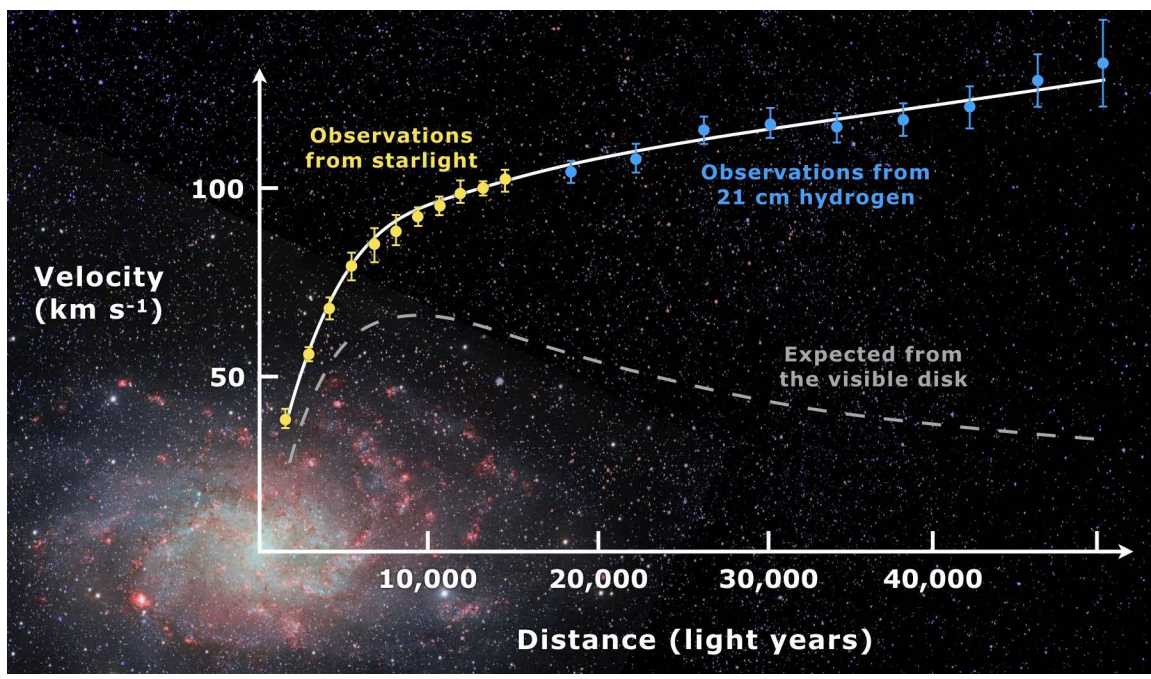

Figure 2. Velocity trend (in $\mathrm{km} / \mathrm{s}$ ) of the stars in the Andromeda galaxy as a function of distance (in thousands of light-years) from the galactic center.

we are thinking that atoms are not stable, but undergo a very slow decay that is reflected in the speed of light emitted.

Then the acceleration corresponding to the shift $\Delta f f$ measured is equal to the sum of two components:

$a_{g}=G M / r^{2}$ due to the rotational motion of the star around the core, and $a_{c}=$ $-H c_{e}$, due to the difference in speed of the light signal emitted by the star at time $t_{e}$ with respect to the speed of the signal emitted by an identical source at time $t_{0}$ of reception. The latter shift is also measured if the gravitational acceleration is zero and the signal sources have constant speed. Therefore

$$
a=G M / r^{2}+\left|a_{c}\right|
$$

The sign of absolute value is used because the direction of $a_{c}$ agrees with the direction of $G M / r^{2}$. Indeed, when we talked about the anomalous acceleration, it was seen that this is directed in the direction of the Earth observer, that is it agrees with the gravitational acceleration.

Summing up, when the gravitational force is negligible because the distance of the star is such that $G M / r^{2} \simeq 0$, the acceleration attributed to the star due to its shift is not zero, but is equal to $a_{c}$.

The relationship between acceleration and speed of the star is $a=v^{2} / r$. It follows

$$
v^{2} / r=G M / r^{2}+\left|a_{c}\right|
$$

Or:

$$
v=\sqrt{\frac{G M}{r}+r\left|a_{c}\right|}
$$

\section{Tully-Fisher Empirical Relationship}

Contrary to the case where $a_{c}=0$, the velocity curve provided by relation (2) has 
a minimum.

After this minimum the trend is parabolic (axis of the parabola parallel to $r$ ), consistent with the trend observed for the galaxy in Figure 2. To verify this relation we use the data relating to the Andromeda galaxy provided by the diagram in Figure 2. From this diagram we can derive the acceleration $a=v^{2} / r$. The graph of Figure 3 is obtained.

The graph shows that the acceleration modulus has a maximum at a critical value of the distance $r_{\text {crit }}=0.8 \times 10^{20} \mathrm{~m}$.

What does this value correspond to? It has been said that $v=\sqrt{\frac{G M}{r}+r\left|a_{c}\right|}$.

The distance $r$ can then be divided into two bands. The one in which the measured shift is due to the rotational motion and therefore to the term $G M / r$, and the next band in which the measured shift is due to the acceleration $a_{c}$.

In the first band, the relationship between speed and acceleration is $v=\sqrt{\frac{G M}{r}}$ and the graph of $v$ as a function of $r$ has a negative slope. In the second band, $v=\sqrt{r\left|a_{c}\right|}$ and the trend of $v$ as a function of $r$ has a positive slope (the trend is parabolic with a horizontal axis and is shown in Figure 2).

The acceleration will therefore resets to zero at the point of separation of the two bands that is obtained when $G M / r=r\left|a_{c}\right|$. From this

$$
r_{\text {crit }}=\sqrt{\frac{G M}{\left|a_{c}\right|}}
$$

The preceding shows that the critical distance value, beyond which the galactic velocity curve remains constant, depends on the baryonic matter $M$ contained in the galaxy. This is consistent with Tully-Fisher's empirical relationship between the intrinsic brightness (proportional to the total mass of visible stars) of a spiral galaxy and the asymptotic value of the velocity curve.

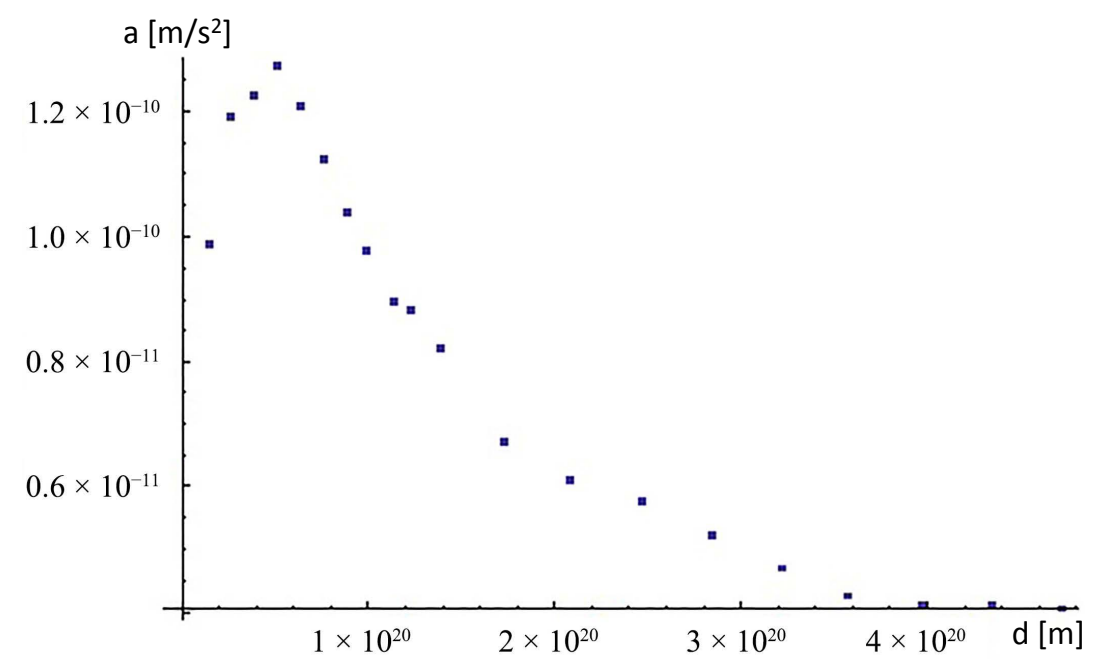

Figure 3. Acceleration trend (in $\mathrm{m} / \mathrm{s}^{2}$ ) of the stars in the Andromeda galaxy as a function of distance (in meters) from the galactic center. 
The relationship discovered by Tully-Fisher links the baryonic mass of the galaxy to the asymptotic value of the velocity curve (indicated with $V_{\text {crit }}$ ) according to a trend of the type $M \propto v_{\text {crit }}^{4}$.

This relationship agrees with the relationship provided by the MOND theory.

However, in VSLT theory, formula (1) receives a more satisfactory theoretical explanation than the one provided by Milgrom. In fact, by substituting

$r_{\text {crit }}=\sqrt{G M / a_{c}}$ in the $v=\sqrt{\frac{G M}{r}+r\left|a_{c}\right|}$ we obtain

$$
v_{\text {crit }}^{4}=\left(4 a_{c}\right) G M
$$

It is clear, however, that the value attributed by Milgrom to the constant $a_{0}$ is not compatible with the value that the VSLT theory attributes to the constant $a_{c}$.

In fact while the constant $a_{c}=\mathrm{d} c / \mathrm{d} t$ is a universal constant that depends only on the time of observation, the constant $a_{0}=v_{\text {crit }}^{4} / G M$ which gives the ratio between the fourth power of the critical velocity of a galaxy and the GM product, depends on the type of galaxy. Figure 4 clearly indicates that one has $a_{0}^{\prime}=2 \times 10^{-10} \mathrm{~m} \cdot \mathrm{s}^{-2}$ for spiral galaxies, while one has $a_{0}^{\prime \prime}=24 \times 10^{-10} \mathrm{~m} \cdot \mathrm{s}^{-2}$ for lenticular galaxies.

The same relation (4) has been derived for galaxies whose mass is concentrated mainly at the center, and it is not sure that all galaxy types meet this condition. For galaxies with mass distribution different from the above one the relation between $a_{c}$ and $a_{0}$ will not be at all $a_{0}=4 a_{c}$ as it happens for galaxies with a super-massive black hole in the center. Milgrom deduces the value of $a_{0}$ from the curve of Figure 1, referring to spiral galaxies, from which, using the interpolating

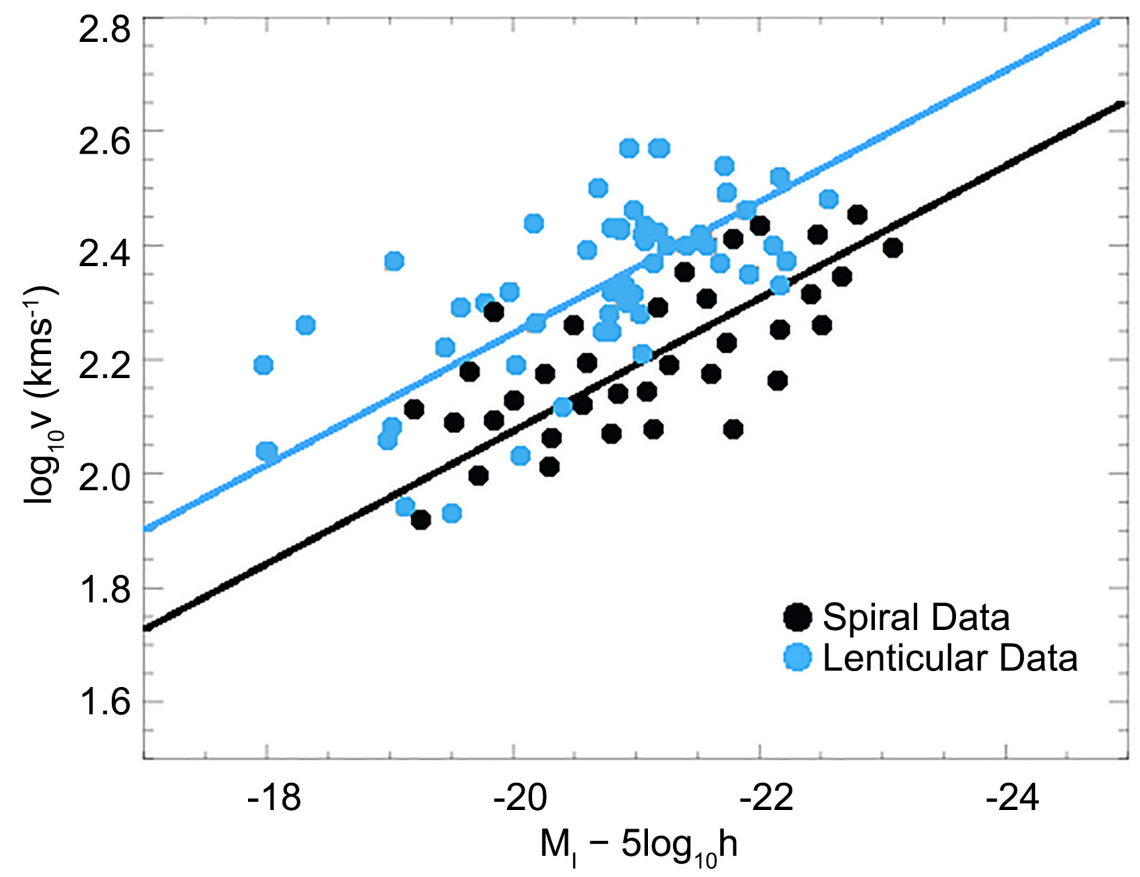

Figure 4. Asymptotic velocity of galaxies (in $\mathrm{km} / \mathrm{s}$ on a logarithmic scale) as a function of luminosity. The latter is assumed proportional to mass. 
line shown, results $\frac{v^{4}}{M}=G a_{0}=1.4 \times 10^{-20}\left(\mathrm{~m}^{4} \cdot \mathrm{sec}^{-4} \cdot \mathrm{kg}^{-1}\right)$. From which $a_{0}=2 \times 10^{-10} \mathrm{~m} / \mathrm{sec}^{2}$.

McGaugh [6] showed that lenticular galaxies have an interpolating line parallel to the line relative to spiral galaxies (and therefore such that $v_{\text {crit }}^{4} \propto M$ ) as Figure 4 indicates.

Using the interpolating line relative to the lenticular galaxies we find

$$
a_{0} G=\frac{v_{c}^{4}}{M}=1.6 \times 10^{-19}\left(\mathrm{~m}^{4} \cdot \mathrm{s}^{-4} \cdot \mathrm{kg}^{-1}\right)
$$

From which $a_{0}=24 \times 10^{-10} \mathrm{~m} \cdot \mathrm{s}^{-2}$. From (4) then follows $a_{c}=6 \times 10^{-10} \mathrm{~m} \cdot \mathrm{s}^{-2}$. This almost is the same value calculated from $\left|a_{c}\right|=H c$, with $H=2.3 \times 10^{-18}$ $\mathrm{s}^{-1}, c=3 \times 10^{8} \mathrm{~m} \cdot \mathrm{s}^{-1}$.

This means that for lenticular galaxies is correct the model assumed previous$1 y$ (mass essentially concentrated in the center of the galaxy), while for spiral galaxies this model is not correct.

Both the Tully-Fisher relationship and Milgrom's hypothesis, have strong experimental foundations but no satisfactory theoretical foundation.

VSLT theory, on the other hand, is capable, as we have seen, to easily explain the experimental relationship of Tully-Fisher. Furthermore, the definition of critical velocity is specified: velocity at the maximum point of the graph of acceleration as a function of distance. It should be noted in fact that, in proximity of $r_{c r i t}$, the speed-distance curve has a parabolic trend with a horizontal axis, and it is not easy, using this graph, to establish what is meant by the asymptotic value of the speed.

Finally, the constant $a_{0}=4 a_{c}$ which in Milgrom is mysterious, acquires a precise meaning in the VSLT theory. That is, it is a multiple of constant $\boldsymbol{a}_{c}$ which expresses the deceleration of light with time.

The value of $a_{c}$ is not deduced from the velocity curves as it happens in the MOND, but it depends on two fundamental parameters such as the Hubble constant and the speed of light.

\section{Acceleration of the Expansion of the Universe}

For galaxies not too far away, $c \simeq c_{0}$ can be assumed, being $c_{0}$ the current value of the speed of light. That is $c_{0} \cong 3 \times 10^{8} \mathrm{~m} / \mathrm{sec}$. From the relation $\mathrm{d} c / \mathrm{d} t=-H c$ we have $\mathrm{d} c l c=-H \mathrm{~d} t$.

By integrating between the instant of emission $t_{e}$ and the receiving instant $t_{0}$ we have:

$$
\int_{t_{e}}^{t_{0}} \frac{\mathrm{d} c}{c}=-H \int_{t_{e}}^{t_{0}} \mathrm{~d} t ; \log c\left(t_{0}\right)-\log c\left(t_{e}\right)=-H\left(t_{0}-t_{e}\right) ; \quad c_{0}=c_{e} \mathrm{e}^{-H\left(t_{0}-t_{e}\right)}
$$

being $c_{0}=c\left(t_{0}\right), c_{e}=c\left(t_{e}\right)$.

If we assume that during the path $r$ traveled by light between the source and the observer, the speed of light remains equal to $c_{e}$ we have $\left(t_{0}-t_{e}\right)=r / c_{e}$. Then 


$$
c_{0}=c_{e} \mathrm{e}^{-\frac{H r}{c_{e}}}
$$

From the previous one we get

$$
c_{e}=\frac{H r}{\text { ProductLog }\left[\frac{H r}{c_{0}}\right]}
$$

The speed of an electromagnetic signal is the product of frequency by wavelength. A change in speed over time can be attributed to a change in frequency or to a change in wavelength. Since the frequency is related to the energy carried by the signal $(E=h f)$ and since it is reasonable to attribute the conservativeness to energy, we attribute the variation of $c$ to a variation of the wavelength emitted by the atoms in different epochs of time.

That is, if $c_{e}, \lambda_{e}$ are speed and wavelength at instant $t_{e}$, and $c_{0}, \lambda_{0}$ are those at instant $t_{0}$, we have

$$
c_{e} / c_{0}=\lambda_{e} / \lambda_{0}
$$

The redshift is defined by the relationship $z=\frac{\lambda_{\text {observed }}-\lambda_{\text {source }}}{\lambda_{\text {source }}}$ where $\lambda_{\text {observed }}$ is the wavelength of the observed signal, coming from a distant source and therefore emitted at time $t_{e}$.

This wavelength will be indicated with $\lambda_{e}$. The $\lambda_{\text {source }}$ is instead the wavelength of the signal emitted by a source NEAR the observer at the instant $t_{0}$, which we indicate with $\lambda_{0}$.

Then

$$
z \equiv \frac{\lambda_{e}-\lambda_{0}}{\lambda_{0}}
$$

From (6) we have

$$
Z=\frac{\lambda_{e}}{\lambda_{0}}-1=\frac{c_{e}}{c_{0}}-1=\frac{\left(\frac{H}{c_{0}}\right) r}{\operatorname{ProdLog}\left(\frac{H}{c_{0}}\right) r}-1
$$

By positing $R_{0}=\mathcal{c}_{0} / H$ the preceding is written:

$$
z=\frac{\frac{r}{R_{0}}}{\operatorname{ProdLog}\left(\frac{r}{R_{0}}\right)}-1
$$

We have $R_{0}=\frac{3 \times 10^{8}}{2.3 \times 10^{-18}}=1.3 \times 10^{26} \mathrm{~m}=13.7 \times 10^{9}$ light-years .

The function ProdLog $(x)$ is defined as follows $x=y \mathrm{e}^{y} \Rightarrow y=\operatorname{ProdLog}(x)$. This function is developable in Taylor series near the origin as follows:

$$
\operatorname{ProdLog}(x) \simeq x-x^{2}+3 x^{3}-\cdots
$$

Assuming $H=69 \mathrm{~km} \cdot \mathrm{sec}^{-1} \cdot \mathrm{Mpc}^{-1}$ we obtain the graph of Figure 5, where the 


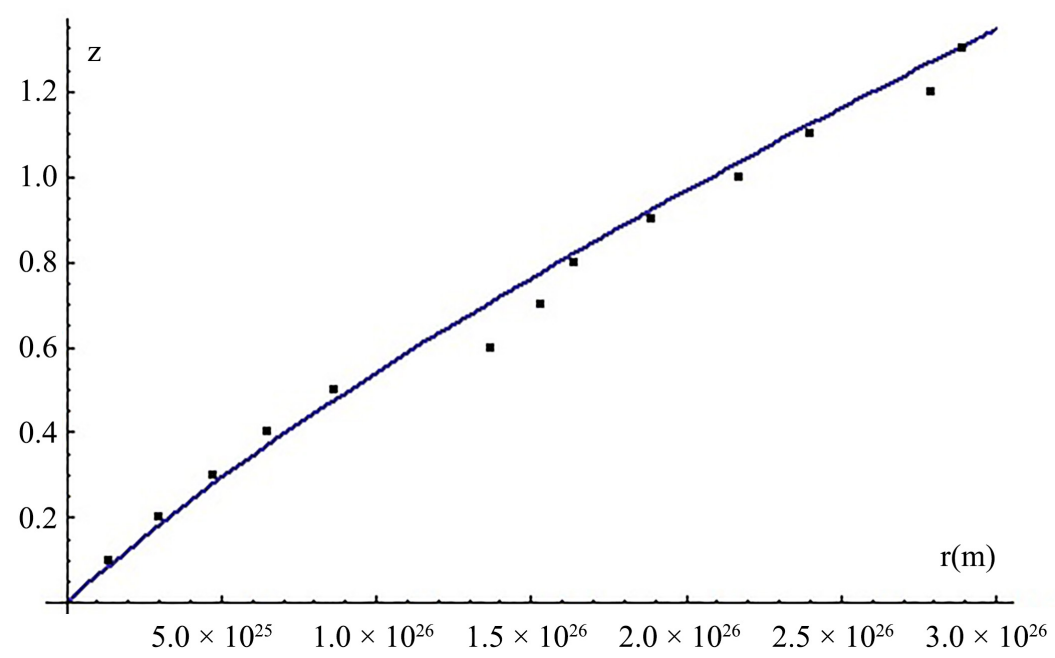

Figure 5. Trend of redshift $z$ as a function of distance (in meters). Points indicate experimental values of 13 galaxies selected in order to interpolate the many available data.

points represent experimental values. As we can see, the theoretical curve giving $z=z(r)$ according to VSLT theory, approximates the experimental values well. The VSLT theory explains therefore the apparent acceleration of the expansion of the universe with time without having to resort to a phantom vacuum energy.

In VSLT theory the expansion of the universe is only apparent, because, as mentioned above, the cosmological redshift is not attributable to a relative removal of galaxies between them, but only to a decrease in the speed of light with time. The distances of galaxies between them are considered constant, since all galaxies are considered in rotational motion around a central point (in which there is a supermassive black hole). Both mass of galaxies and black hole are considered constant in time, so it is constant also the distance of each galaxy from the black hole, and the average distance between them. It is the rotation around the nucleus that prevents galaxies in the universe to collapse because of gravitational force.

Only a rotational motion can allow the stability of a system of masses between which acts an attractive force as the gravitational force.

Therefore, the so-called "Cosmic Microwave Background" (CMB), according to VSLT, is originated by some mechanism different from the Big Bang. For example by the absorption and subsequent re-emission in random direction, by the cosmic dust, of the radiation emitted by stellar sources. The temperature of this radiation would be then $2.7^{\circ} \mathrm{K}$, which is the temperature of deep space.

\section{The Flatness Problem}

From Equation (5) $c_{e}=\frac{c_{0} \frac{r}{R_{0}}}{\operatorname{ProductLog}\left(\frac{r}{R_{0}}\right)}$.

We wonder what the physical meaning of the constant $R_{0}=c_{0} / H$ might be. 
The relation $v_{\text {lim }}^{4}=4\left|a_{c}\right| G M$ which we have seen above, binds the limit velocity (assumed by the most distant stars in the galaxy) to the galactic mass. This limit velocity increases with the increase of $M$. The maximum value that the limit velocity can assume (at the present time) is $c_{0}$. Therefore the mass of the universe, assuming that the farthest stars had this speed, would be $M=\frac{c_{0}^{4}}{4\left|a_{c}\right| G}=4.3 \times 10^{-27} \mathrm{~kg}$. If the radius of the universe was just $R_{0}$ the density of matter that would be found would be

$$
\rho=\frac{M}{(4 / 3) \pi R_{0}^{3}}=4.6 \times 10^{-27} \mathrm{~kg} / \mathrm{m}^{3}
$$

In fact, if we substitute the relation found for $M$ into the relation for $\rho$, we get: $\rho=\frac{\frac{c_{0}^{4}}{4\left|a_{c}\right| G}}{(4 / 3) \pi R_{0}^{3}}$.

If we refer to the current time we get: $\rho_{0}=\frac{\frac{c_{0}^{4}}{4\left|a_{c 0}\right| G}}{(4 / 3) \pi R_{0}^{3}}$.

Being $a_{c 0}=\left(\frac{\mathrm{d} c}{\mathrm{~d} t}\right)_{t=t_{0}}$, and $\frac{\mathrm{d} c}{\mathrm{~d} t}=-H c$, we have $\left|a_{c 0}\right|=H c_{0}$. Then $\rho_{0}=\frac{\frac{c_{0}^{4}}{4 H c_{0} G}}{\left(\frac{4}{3}\right) \pi \frac{c_{0}^{3}}{H^{3}}}=\frac{c_{0}^{4}}{4 H c_{0} G} \frac{3 H^{3}}{4 \pi c_{0}^{3}}$

$$
\rho_{0}=\frac{3 H^{2}}{16 \pi G}
$$

This value is just half of the value that RG indicates as critical mass, that is the value that should have the density mass of the universe for its geometry to be flat. In RG this raises a problem known as "flatness problem". That is several measures indicate that geometry of universe is flat and yet density of visible matter is much lower than critical value (hence the need to assume the existence of dark matter).

In VSLT there is no missing mass problem since (9) does not represent a half of the density that the universe should have for its geometry to be flat. But it simply represents the maximum possible density, obtained under the assumption that its peripheral galaxies rotate around the black hole at the center of the universe (Great Attractor) with velocity $v_{c r i t}=c_{0}$. The fact that a much lower density than the critical one is observed simply means that the velocity of such galaxies is much lower than $c_{0}$.

\section{VSLT Theory and General Relativity}

Let's see how Relativity explains the cosmological redshift phenomenon. Suppose that a light pulse is emitted by a source along the $r$ direction that joins the source and the observer. 
The simplest relativistic model predicts a homogeneous and isotropic universe, with flat geometry. This model is called the flat FRW model. The homogeneity and symmetry of the universe on a large scale are confirmed by astronomical observations. The flatness of the universe is a datum relative to its curvature. There are three possible types of curvature compatible with the assumption of homogeneity and symmetry. That is, geometry with zero, positive, or negative curvature. The three types of curvature are related to the density of matter observed for the universe. If the observed density is equal to a theoretical density $\rho_{\text {crit }}=3 H_{0}^{2} /(8 \pi G)$ then the geometry of the universe is flat, if it is greater than the critical one the geometry is spherical, finally if $\rho<\rho_{\text {crit }}$ the geometry is hyperbolic.

Observational data are indicating a flat geometry.

In this hypothesis, the line element of spacetime, in Cartesian coordinates $x, y, z$ is:

$$
\mathrm{d} s^{2}=-\mathrm{d} t^{2}+a(t)\left[\mathrm{d} x^{2}+\mathrm{d} y^{2}+\mathrm{dz} z^{2}\right]
$$

The previous one was written in a system where $c=c_{0}=1$. In fact, for relativity $c$ is an absolutely constant factor. It can therefore be thought as a conversion factor between the unit of measurement of space (chosen arbitrarily) and that of time (also arbitrary). We can therefore set this factor equal to 1 and in this way the unit of measurement of space and that of time coincide. Resulting with this choice $s=t$, also the time will be measured in meters.

In the above $a(t)$ is a dimensionless function of time called scale factor.

In the polar coordinates $r, \theta, \varphi$ the previous one is written:

$$
\mathrm{d} s^{2}=-\mathrm{d} t^{2}+a^{2}(t)\left[\mathrm{d} r^{2}+r^{2} \mathrm{~d} \theta^{2}+r^{2} \sin ^{2} \theta \mathrm{d} \varphi^{2}\right]
$$

Let us now consider a beam of light that travels along the $r$ direction, directed towards the observer placed in the origin of the coordinates.

For this beam we have $\mathrm{d} s^{2}=-\mathrm{d} t^{2}+a^{2}(t) \mathrm{d} r^{2}$.

Light travels along world lines with a null line element, or $\mathrm{d} s=0$.

Follows $0=-\mathrm{d} t^{2}+a^{2}(t) \mathrm{d} r^{2}$ that is $\mathrm{d} r=\frac{\mathrm{d} t}{a(t)}$.

We can interpret the relationship between the space element $\mathrm{d} r$ traveled by light, referring to the time element $\mathrm{d} t$ needed to travel it, just like the speed of light.

Therefore the link between $c(t)$ in the interpretation $c$ variable, and $a(t)$ in the relativistic interpretation with constant $c$ and a variable, is $c(t)=1 / a(t)$. Or

$$
a(t)=\frac{1}{c(t)}
$$

From the previous relation, if $c$ is function of the time:

$$
\frac{\mathrm{d} a}{\mathrm{~d} t}=-\frac{1}{c^{2}} \frac{\mathrm{d} c}{\mathrm{~d} t} ; \frac{\mathrm{d} a / \mathrm{d} t}{a(t)}=\frac{-\frac{1}{c^{2}} \frac{\mathrm{d} c}{\mathrm{~d} t}}{\frac{1}{c}}=H ;
$$




$$
\frac{\mathrm{d} c}{\mathrm{~d} t}=-H c
$$

This is the foundational relationship of VSLT theory.

This formula is demonstrated in the paper of Jef Van Royen [7], starting from the Friedman equation for an expanding homogeneous and isotropic universe. In that paper it is shown how the distance covered by a beam of light with radial direction can be obtained by assigning to the light a time-varying velocity equal to $c(t)=\left(a_{0} c_{0}\right) / a(t)$. This relation is substituted into the Friedman equation and it is shown that the solutions obtained are identical to those obtained under the hypothesis c constant. Van Royen shows how the VSL hypothesis can solve important problems such as horizon or flatness without having to resort to the inflationary hypothesis. However, it should be pointed out that the hypothesis of present paper is profoundly different from the VSL hypothesis advocated by several authors [8], [9] to solve some problems in relativistic cosmology without having to resort to inflation.

In fact in VSL optics the speed of light varies during the path from the source to the observer and this variation is due to the expansion of space as it happens in relativistic optics.

Vice versa VSLT theory predicts a variation of $c$ with time. That is it provides that the same source, with time variation, emits photons at different speed. Once emitted photon travel in empty space at the same speed with which it was emitted. Its frequency, during the flight from source to observer does not vary. Therefore it is not necessary to provide any mechanism to explain the variation between emission frequency and observed one, because this variation does not occur during this journey but it is due to the difference between emission time and observation time.

\section{Cosmological Redshift in General Relativity}

We indicate with $t_{e}$ the instant of emission of a light pulse and with $t_{0}$ the instant of detection. During the interval $t_{0}-t_{\mathrm{e}}$ the light has traveled the distance R. So $\int_{0}^{R} \mathrm{~d} r=R=\int_{t_{e}}^{t_{0}} \frac{\mathrm{d} t}{a(t)}$.

Suppose that the source emits a second pulse at the instant $t_{e}+\delta t_{e}$. It will be received instantly $t_{0}+\delta t_{0}$. Since this second impulse also travels the distance $R$, it will be:

$$
R=\int_{t_{e}}^{t_{0}} \frac{\mathrm{d} t}{a(t)}=\int_{t_{e}+\delta t_{e}}^{t_{0}+\delta t_{0}} \frac{\mathrm{d} t}{a(t)}
$$

Let us consider the graph of the integrand function $1 / a(t)$. Let $A$ be the area subtended by this graph between the points $t_{e}$ and $t_{e}+\delta t_{e}$. Let B be the area between $t_{e}+\delta t_{e}$ and $t_{0}$, and let $C$ be the area between $t_{0}$ and $t_{0}+\delta t_{0}$. The above relationship means $A+B=B+C$. That is $A=C$. But the area $A$ is equal to $\delta t_{e} / a\left(t_{e}\right)$, while the area $C$ is equal to $\delta t_{0} / a\left(t_{0}\right)$. 
Then $\frac{\delta t_{e}}{a\left(t_{e}\right)}=\frac{\delta t_{0}}{a\left(t_{0}\right)}$.

If instead of just two pulses is generated a train of pulses spaced by the interval $\delta t_{e}$ the frequency of this train will be $f_{e}=1 / \delta t_{e}$, while the frequency of the received pulse train will be $f_{0}=1 / \delta t_{0}$. Therefore

$$
\begin{gathered}
\frac{1}{f_{e}} * \frac{1}{a\left(t_{e}\right)}=\frac{1}{f_{0}} * \frac{1}{a\left(t_{0}\right)} . \\
f_{0}=f_{e} \frac{a_{e}}{a_{0}}
\end{gathered}
$$

having set $a_{e}=a\left(t_{e}\right), a_{0}=a\left(t_{0}\right)$.

The previous formula is the basis of HUBBLE'S LAW according to the relativistic model.

That is, the frequency of a signal measured at time $t_{0}$ is different from the frequency $f_{e}$ of the same signal emitted at time $t_{e}$ because the scale factor $a(t)$ has varied between the two instants $t_{e}$ and $t_{0}$.

In particular, since it is assumed that the scale factor $a(t)$ increases with the passage of time (expansion of the universe), we have $a_{0}>a_{e}$ and therefore $f_{0}<f_{e}$.

For a light signal we have $c=\lambda f$. Since in Relativity $c$ is considered constant we have $\lambda_{0} f_{0}=\lambda_{e} f_{e}$.

So $f_{0} / f_{e}=\lambda_{e} / \lambda_{0}$. Then $\lambda_{e} / \lambda_{0}=a_{e} / a_{0} ; \frac{\lambda_{0}}{\lambda_{e}}-1=\frac{a_{0}}{a_{e}}-1$.

The ratio $z \equiv \frac{\lambda_{\text {observed }}-\lambda_{\text {source }}}{\lambda_{\text {source }}}$ it is the cosmological redshift. Then: $z=\frac{\lambda_{0}}{\lambda_{e}}-1=\frac{f_{e}}{f_{0}}-1$. That is

$$
z+1=\frac{a_{0}}{a_{e}}
$$

We develop the Taylor series function $a(t)$ around a point $t_{0}$.

$$
a(t)=a\left(t_{0}\right)+\left(t-t_{0}\right)\left(\frac{\mathrm{d} a}{\mathrm{~d} t}\right)_{t_{0}}+\frac{\left(t-t_{0}\right)^{2}}{2}\left(\frac{\mathrm{d}^{2} a}{\mathrm{~d} t^{2}}\right)_{t_{0}}+\cdots
$$

If we suppose that $a(t)$ is slowly variable in time, we can neglect the higher order terms than the first, obtaining:

$$
a(t)=a\left(t_{0}\right)+\left(t-t_{0}\right)\left(\frac{\mathrm{d} a}{\mathrm{~d} t}\right)_{t_{0}}
$$

We apply the previous one to the instant of emission of a light beam, obtaining:

$$
a_{e}=a_{0}+\left(t_{e}-t_{0}\right) \dot{a}_{0}
$$

If by $t_{0}$ we mean the current time, the difference $\left(t_{0}-t_{e}\right)$ is equivalent to the DISTANCE traveled by the light beam divided by the emission speed $c_{e}$. That is $\left(t_{0}-t_{e}\right)=d / c_{e}$ so

$$
a_{0}-a_{e}=\left(t_{0}-t_{e}\right) \dot{a}_{0}=\frac{d}{c_{e}} \dot{a}_{0}
$$




$$
\begin{gathered}
\frac{a_{0}-a_{e}}{a_{e}}=\left(t_{0}-t_{e}\right) \dot{a}_{0}=\frac{d}{c_{e}} \frac{\dot{a}_{0}}{a_{e}}=\frac{d}{c_{e}} \frac{\dot{a}_{0}}{a_{e}} \frac{a_{0}}{a_{0}} \\
z=\frac{d}{c_{e}} \frac{\dot{a}_{0}}{a_{0}}(z+1)
\end{gathered}
$$

In the case of small distances we have $c_{e}=c_{0}, \quad z \ll 1$, therefore

$$
z=z_{0}=\frac{\dot{a}_{0} / a_{0}}{c_{0}} d
$$

The above is equivalent to Hubble's law if

$$
\frac{\dot{a}_{0}}{a_{0}}=H_{0}
$$

It should therefore be noted that in relativity the Hubble constant is not a universal constant, but its value depends on the observation time.

\section{Light Curves of Type Ia Supernovae}

An argument correctly proposed against the theory of "tired light" which attributes the redshift to the decrease in the speed of light during the journey from the source to the observer, is relating to the widening of the light curves of type Ia Supernovae.

These appear as stars of the first magnitude whose existence, however, is of the order of a few months. If we draw a diagram of the luminosity of these supernovae as a function of time (light curves) we find that for some types of supernovae, indicated by the abbreviation $\mathrm{SNela}$, this diagram has a characteristic shape in which there is a first rapidly increasing phase and a second phase slowly decreasing. The slope of the descending phase is in relation to the distance of such sources from the Earth. This relationship has been verified with a high degree of precision to the point that previous supernovae have assumed the role of "standard candles" for the measurement of galactic distances. It is verified that the life of a supernova, or the width of its light curve, undergoes an enlargement, as its redshift $\mathrm{z}$ increases, according to the relation:

$$
w=1+z
$$

In the previous one, $w$ is the ratio between the width of the light curve for a supernova at distance $z$ and the width of the light curve for a supernova at distance $z_{0} \ll 1$.

The graph in Figure 6, due to Goldhaber, provides experimental proof of this relation.

The figure shows that tired light theory should produce a widening factor $\mathrm{w}$ equal to 1 for each value of $z$ (horizontal curve in the figure). Indeed this theory does not contemplate any variation of the speed of light with the time of emission, but only with the distance crossed by the light signal.

Let be $t_{1}$ the instant in which the supernova birth event occurs and $t_{2}$ the instant in which the supernova death occurs. The birth will be perceived by the terrestrial observer at the instant $t_{1}^{\prime}$ while the death will be perceived at the instant $t_{2}^{\prime}$. 


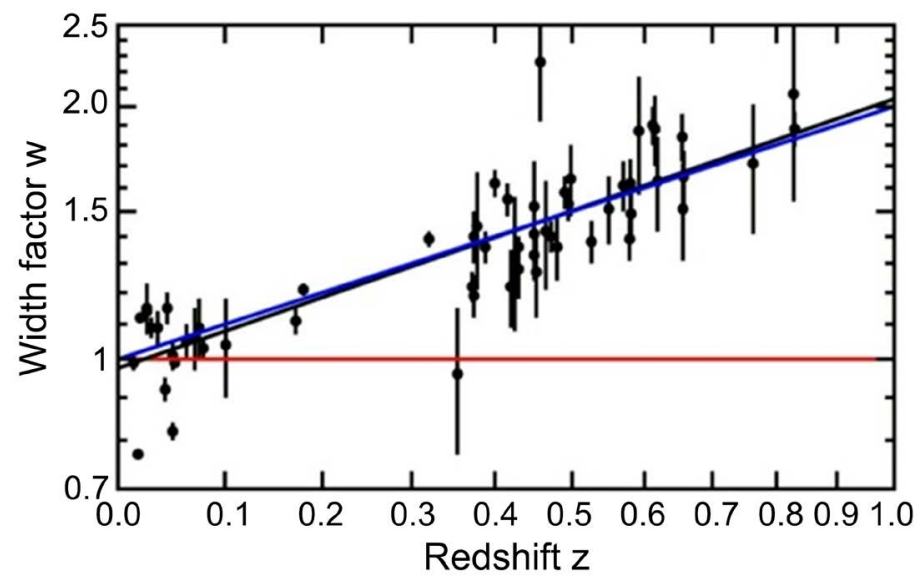

Figure 6. Light curve broadening factor of type 1a supernovae as a function of their redshift.

We have $t_{1}^{\prime}=t_{1}+d / c_{\text {med }}$, being $d$ the distance of the source from the observer and $c_{\text {med }}$ an average value of the speed of light during the path $\mathrm{d}$ (the theory of tired light assumes that $c$ varies during this path). It will be $t_{2}^{\prime}=t_{2}+d / c_{\text {med }}$. The average speed $c_{m e d}$ in the two cases is the same since the distance traveled by the light is the same. Then it would turn out $t_{2}^{\prime}-t_{1}^{\prime}=t_{2}-t_{1}$.

This does not happen for the VSLT theory which, unlike the tired light theory, predicts that the same source at different times, produces a light with different speed. We have seen that $z=\left(c_{e}-c_{0}\right) / c_{0}$, hence $c_{e}=c_{0}(z+1)$.

Suppose there is a source $S$ at a distance $R$ from the Earth. If a signal is emitted at time $t_{e}$ when the speed of light is $c_{e}$ it will be detected at time $t_{e}^{\prime}=t_{e}+R / c_{e}$. If the signal is emitted at the instant $t_{0}$ when the speed is $c_{0}$, it will be revealed at the time $t_{0}^{\prime}=t_{0}+R / c_{0}$.

Obtaining $c_{e}, c_{0}$ from these formulas and substituting in $z=\left(c_{e}-c_{0}\right) / c_{0}$ we get $R / t_{e}=\left(R / t_{0}\right)(1+z)$, that is $t_{0}=t_{e}(1+z)$. Then

$$
\Delta t_{0}=(1+z) \Delta t_{e}
$$

\section{Relativistic Interpretation versus VSLT Interpretation}

Also General Relativity provides for the light curve of supernovae an enlargement factor w equal to $1+z$.

Indeed, for (12), $z+1=a_{0} / a_{e}$. The light curve of supernovae widens as the distance increases because between the moments of birth and death of the supernova the distance, measured by the scale coefficient $a(t)$, increases.

In intuitive terms, when the supernova is born at the instant $t_{1}$ the distance is $r_{1}$, and the time in which the signal reaches the observer is $t_{1}+r_{1} / c$. When the supernova dies at the instant $t_{2}$, the distance is $r_{2}$ and the time in which the signal reaches the observer is $t_{2}+r_{2} / c$.

So the life of the supernova seen by the observer is $t_{2}-t_{1}+\left(r_{2}-r_{1}\right) / c$.

The enlargement is $w=\frac{t_{2}-t_{1}+\left(r_{2}-r_{1}\right) / c}{t_{2}-t_{1}}=1+\frac{\mathrm{d} r / \mathrm{d} t}{c}=1+\frac{\mathrm{d} r / \mathrm{d} t}{r c} r$ 
The scale factor $a(t)$ is proportional to the distance $r(t)$ of a distant galaxy from the Earth.

Hence $\frac{\mathrm{d} r / \mathrm{d} t}{r c}=\frac{\mathrm{d} a / \mathrm{d} t}{a c}$. It follows $\frac{\mathrm{d} a / \mathrm{d} t}{a c} r=\frac{H}{c} r=z$. Then $w=z+1$ also for relativity.

The difference between the relativistic interpretation and the VSLT interpretation of various phenomena such as the cosmological redshift, the critical density, the widening of the light curves of supernovae, and so on, is the following.

The speed of light can be defined as the ratio between an increase in space and an increase in time.

$$
c=\Delta l / \Delta t
$$

To measure the speed of light it is therefore necessary to define a unit of measurement for space and a unit of measurement for time.

To measure the space, a macroscopic sample (such as a platinum-iridium bar maintained at a constant temperature, or a microscopic sample (for example the wavelength of a specific spectral line) can be used. Similarly, to measure the time it can be used a macroscopic sample (period of rotation of the Earth) or a microscopic sample (inverse of the frequency of a given spectral line).

The VSLT theory believes that the relationship between the speed of a body measured with macroscopic instruments, and that measured with microscopic instruments varies over time.

For example, if I measure the time interval between the outward and return journey of a beam of light between the two notches of a platinum-iridium bar maintained at a constant temperature, this interval will appear to increase over time.

No one can say with certainty that the length of a platinum-iridium bar stored at a constant temperature remains unchanged over time. The relativistic interpretation is that if you measure an increase in the round trip time of a light beam between the two ends of a bar, this fact must necessarily be attributed to an elongation of the bar. The interpretation of the VSLT is instead that, if no reason is discovered why this length should vary, and if the increase in the round trip time of the light beam occurs for each bar, it must be attributed to a variation of $c$.

The hypothesis $c$ variable in time has important theoretical implications. If we use the relativistic postulate of constancy of the speed of light we must attribute to photons a zero mass. But this prevents masses to exert a gravitational force on photons (gravitational forces are proportional to the product of masses). Therefore the only way we have to explain the deviation of photons trajectory by a mass $M$ is to assume that $M$ deforms the space. VSLT theory assumes instead that $c$ can vary and then the photon has a mass, however small. Under this assumption the problems related to the curvature of the universe disappear. Space is flat and photons can curve their trajectory because they are subjected to gravitational forces. 
VSLT theory introduces a universal constant $R_{0}=c_{0} / H$. It is likely that this constant enters into the gravitational force formula that needs to be modified to properly account for relativistic effects such as Mercury's perihelion precession or the deflection of light from distant sources by the Sun. The gravitational potential created by a mass $M$ at distance $r$ could be

$$
\Phi(r)=-\frac{G M \mathrm{e}^{-\frac{r}{R_{0}}}}{r}=-\frac{G M \mathrm{e}^{-\mu r}}{r}
$$

The above formula is identical to the form of the potential that Yukawa gives to nuclear forces, which are forces with limited range of action. Yukawa calculates for the action quanta of forces with potential of the predicted type, a mass $m=\mu \hbar / c$ which in our case becomes $m=\hbar /\left(R_{0} c\right)$. This value turns out to be extremely small. This would explain the weakness of the gravitational forces.

The photon would be then the action quantum not only of electric forces but also of gravitational forces and this fact should lead to a dependence of $G$ on the constant $\mathcal{C}$, in analogy to what happens for the constant $\mathrm{K}$ that appears in Coulomb's law.

\section{Conclusions}

There is no contradiction between the theory of General Relativity and VSLT theory that assumes $c$ variable in time. All laws valid in the first theory are valid in the second and vice versa.

To clarify the difference between the two points of view consider an observer attached to a carousel rotating with constant angular velocity, he observes each object remaining on the land as if it were rotating around a point $P$.

He can think that he is stationary and that each object he observes (and of which he can calculate with precision the trajectory) is rotating around a point $\mathrm{P}$ (different for each object).

Or he can think that he is moving with respect to a reference $R$, and that the objects that he observes are stationary with respect to $R$.

The two choices are both theoretically valid, since a scientific theory is valid if it is consistent and if it is able to make correct predictions. However, in the first hypothesis the observer will conclude that at the point $P$ there is some mass that causes the rotation of the object around $P$ and, since he does not observe any mass in $P$, he will conclude that it is made of "dark matter".

For the sake of completeness, it is necessary to say that the cited dark matter and Universe's accelerated expansion issues can be, in principle, achieved in the framework of extended gravity. This is stressed, for example, in [10].

\section{Acknowledgements}

The author thanks Prof. Jef Van Royen for providing a derivation of the relationship on which this paper is based, albeit from a different perspective than that of the author. Grateful thanks go to my friend Domenico Militano for his 
numerous and useful comments on the topics covered.

\section{Conflicts of Interest}

The author declares no conflicts of interest regarding the publication of this paper.

\section{References}

[1] Pipino, G. (2019) Evidences for Varying Speed of Light with Time. Journal of High Energy Physics, Gravitatation and Cosmology, 5, 395-411.

[2] Anderson, J.D. (1998) Indication from Pioneer 10/11, Galileo, and Ulysses Data, of an Apparent Anomalous, Weak, Long Range Acceleration. arXiv:gr-qc/9808081v2

[3] Goldhaber, G. (2001) Timescale Stretch Parameterization of Type Ia Supernova B-Band Light Curves. The Astrophysical Journal, 558.

[4] Milgrom, M. (1983) A Modification of the Newtonian Dynamics as a Possible Alternative to the Hidden Mass Hypothesis. The Astrophysical Journal, 270, 365-370. https://doi.org/10.1086/161130

[5] Tully, R.B. and Fisher, J.R. (1977) A New Method of Determining Distances to Galaxies. Astronomy and Astrophysics, 54, 661-673.

[6] McGaugh, S.S., Schombert, J.M., Bothun, G.D. and de Blok, W.J.G. (2000) The Baryonic Tully-Fisher Relation. The Astrophysical Journal Letters, 533, L99. arXiv:astroph/0003001 https://doi.org/10.1086/312628

[7] Van Royen, J. (2021) General Relativity: Varying Speed of Light from the Friedmann Equation. Journal of Cosmology, 27, 15389-15406.

[8] Albrecht, A. and Magueijo, J. (1998) A Time Varying Speed of Light as a Solution to Cosmological Puzzles. (Preprint) https://doi.org/10.1103/PhysRevD.59.043516

[9] Barrow, J.D. (1998) Cosmologies with Varying Light-Speed. Arxiv:astro-ph/9811022v1

[10] Corda, C. (2009) Interferometric Detection of Gravitational Waves: The Definitive Test for General Relativity. International Journal of Modern Physics D, 18, 2275-2282. https://doi.org/10.1142/S0218271809015904 